\title{
TÉCNICA DE PARAMETRIZAÇÃO GEOMÉTRICA PARA O FLUXO DE CARGA CONTINUADO BASEADO NAS VARIÁVEIS TENSÃO NODAL E FATOR DE CARREGAMENTO
}

\author{
Alfredo Bonini Neto*
}

Dilson Amancio Alves*

*UNESP - Departamento de Engenharia Elétrica,

Av. Brasil, 56, Cx. P. 31, 15385-000 Ilha Solteira - Brasil

\section{RESUMO}

Este trabalho apresenta um novo esquema de parametrização geométrica para o fluxo de carga continuado que possibilita o traçado completo das curvas $\mathrm{P}-\mathrm{V}$, e o cálculo do ponto de máximo carregamento de sistemas de potência, sem os problemas de mau-condicionamento. Esta nova técnica de parametrização geométrica foi obtida a partir da observação das trajetórias de solução do fluxo de carga. A técnica associa robustez com facilidade de compreensão. A singularidade da matriz Jacobiana é eliminada pela adição da equação de uma reta que passa por um ponto no plano formado pelas variáveis magnitude da tensão nodal e o fator de carregamento. $\mathrm{O}$ uso desta técnica amplia o grupo das variáveis de tensão que pode ser usado como parâmetro. Os resultados obtidos com a nova metodologia para sistemas do IEEE (14, 118 e 300 barras), mostram que as características do método convencional são melhoradas e a região de convergência ao redor da singularidade é ampliada. Vários testes são apresentados com intuito de prover um completo entendimento do método proposto e possibilitar comparação e avaliação do desempenho do esquema de parametrização proposto.

PALAVRAS-CHAVE: Método da Continuação, Fluxo de Carga, Múltipla Soluções, Ponto de Máximo Carregamento.

Artigo submetido em 17/08/2007

1a. Revisão em 14/11/2007

2a. Revisão em 12/05/2008

Aceito sob recomendação do Editor Associado Prof. Carlos A. Castro

\section{ABSTRACT}

This work presents a new geometrical parameterization scheme to the continuation power flow that allows the complete tracing of $\mathrm{P}-\mathrm{V}$ curves and the computation of the maximum loading point of a power system, without illconditioning problems. This new geometrical parameterization technique was obtained from the observation of the behavior of power flow solutions path. The technique associates robustness with simplicity and easy interpretation. The Jacobian matrix singularity is overcome by the addition of a line equation, which passes through a point in the plane determined by the bus voltage magnitude and the loading factor variables. The use of this technique enlarges the group of voltage variables that can be used as continuation parameter. The results obtained with the new methodology for the IEEE systems (14, 118 and 300 buses) show that the characteristics of the conventional method are enhanced and the region of convergence around the singular solution is enlarged. Many tests are also presented in intention to provide the complete understanding of the proposed method and to allow the comparison and assessment of the performance of the proposed parameterization scheme.

KEYWORDS: Continuation Method, Load Flow, Multiple solutions, Maximum Loading Point.

\section{INTRODUÇÃO}

Entre os diversos estudos relacionados com o planejamento e a operação de sistemas elétricos, a análise de estabilidade 
estática de tensão tem despertado um grande interesse. Isso se deve ao fato dos sistemas elétricos passarem a operar próximo de seus limites operacionais em consequiência direta do crescimento contínuo da demanda, das restrições econômicas e ambientais, e da desregulamentação do setor elétrico. Para a avaliação da estabilidade de tensão, as empresas do setor elétrico internacional (WSCC, 1998) e nacional (FTCT, 1999) têm recomendado o levantamento do perfil de tensão das barras em função de seu carregamento (curvas P-V e QV). Esta recomendação se deve ao fato de que essas curvas possibilitam a compreensão das condições operativas do sistema em diferentes condições de carregamento e contingências. Estes perfis são utilizados, entre outras, para: determinar os limites de transferência de potência entre as áreas de um sistema; ajustar margens; observar o comportamento das tensões das barras do sistema em análise; e comparar estratégias de planejamento.

Na análise estática de estabilidade de tensão, a obtenção do ponto de máximo carregamento (PMC) do sistema é importante tanto para o cálculo de margens de estabilidade quanto para a realização da análise modal. Uma vez que o PMC define a fronteira entre as regiões de operação estável e instável, é neste ponto que a análise modal fornecerá as informações para a determinação de medidas efetivas para o reforço do sistema.

Tradicionalmente, o levantamento da curva P-V tem sido realizada através da obtenção da solução do fluxo de carga (FC), usando o método de Newton, para sucessivos incrementos do carregamento do sistema. Este procedimento é realizado até que o processo iterativo deixe de convergir. Para fins práticos, este ponto é considerado como sendo o PMC. Entretanto, sabe-se que os problemas de convergência encontrados pelo FC convencional para a obtenção do PMC são conseqüentes das dificuldades numéricas associadas à singularidade da matriz Jacobiana (J). Assim sendo, o uso dos métodos convencionais de FC para a obtenção das curvas P-V fica restrito à sua parte superior (correspondendo a operação estável). Além dos problemas relacionados com a singularidade de J, a solução do FC também dependerá das características comuns aos processos de solução de equações algébricas não-lineares, tais como do método utilizado na resolução, da existência da solução, das múltiplas soluções existentes e da estimativa inicial. Dessa forma, sempre será necessário ponderar se os problemas de não convergência são devidos a problemas numéricos ou a limitações físicas do sistema. Em geral, as diferenças não são óbvias.

Os métodos de fluxo de carga continuados (FCC) superam as dificuldades numéricas acima mencionadas pela adição de equações parametrizadas (Cañizares et al., 1992; Alves et al., 2000, Ajjarapu e Christy, 1992; Chiang et al., 1995; Garbelini et al., 2005, Seydel, 1994). As equações do FC são refor- muladas visando à eliminação da singularidade da matriz $\mathbf{J}$ no PMC e, consequentemente, dos problemas numéricos que ocorrem em torno deste. Assim, o traçado completo do perfil de tensão pode ser efetuado variando automaticamente o valor de um determinado parâmetro do sistema. A diferença entre os métodos de FCC está no modo como o novo parâmetro é escolhido e em como a singularidade é eliminada. As técnicas de parametrização mais utilizadas pelos FCC para eliminar a singularidade de $\mathbf{J}$ são a geométrica (Cañizares et al., 1992; Chiang et al., 1995) e a local (Ajjarapu e Christy, 1992).

Iba et al., (1991) apresentaram uma técnica para contornar a singularidade de $\mathbf{J}$ sem a necessidade de parametrização. Posteriormente essa técnica, associada a um controle de passo, foi aplicada com sucesso em vários sistemas em Cañizares et al. (1992). Chiang et al. (1995) propuseram uma parametrização onde o comprimento de arco $(s)$ é utilizado como parâmetro. Entretanto, como o sistema de equação formado no passo preditor é não linear, a sua solução exige um método especial, o que pode implicar num tempo computacional muito alto. A opção sugerida pelos autores foi o uso do preditor secante logo após a obtenção de dois pontos da curva. De acordo com os autores esta técnica de parametrização é mais robusta, possibilitando que sejam dados maiores passos do que a técnica utilizando parametrização local. Essa técnica de parametrização local (Ajjarapu e Christy, 1992; Seydel 1994) consiste na troca de parâmetro próximo ao ponto de máximo carregamento (PMC). No método do vetor tangente a variável escolhida é aquela que apresentar a maior variação, sendo que o fator de carregamento $(\lambda)$ passa a ser tratado como variável dependente, enquanto que a variável escolhida passa a ser o novo parâmetro.

Uma das vantagens importantes acrescida pelos métodos de FCC é a possibilidade da visualização da geometria da trajetória de solução das equações do FC (curva P-V), a qual é útil não só do ponto de vista didático, posto que esta facilita a compreensão do fenômeno em si, mas também auxilia no desenvolvimento de novas estratégias para a eliminação de problemas numéricos relacionados aos métodos de solução das equações, a obtenção das múltiplas soluções e de índices de estabilidade de tensão (Chen e Wang, 1997; Overbye e Klump, 1996; Yorino et al., 1997).

A técnica de parametrização local proposta em Ajjarapu e Christy (1992) tem demonstrado que ao aproximar-se do PMC, o parâmetro muda de $\lambda$ para a tensão que apresenta a maior variação, retornando novamente para $\lambda$ após alguns pontos. Embora o uso desta técnica para a escolha automática do parâmetro não tenha apresentado dificuldades, o conjunto das barras cuja magnitude de tensão pode ser utilizada como parâmetro da continuação fica muito restrito, particularmente nos sistemas com grande número de barras de ge- 
ração $(P V)$ e OLTC (transformadores com comutação de tap sob carga).

Com o intuito de se esclarecer as dificuldades encontradas na escolha da magnitude de tensão de uma barra como parâmetro, considere a figura 1 , onde se podem ver as curvas $\mathrm{P}-\mathrm{V}$ de algumas barras do sistema IEEE-118, as quais foram obtidas a partir dos métodos apresentados em trabalhos anteriores (Alves, 2000). A curva 1 na figura 1(a) foi obtida desconsiderando a atuação dos tap's (i.e., os tap's são mantidos fixos durante todo o traçado da curva $\mathrm{P}-\mathrm{V}$ ) e das potências reativas das barras $P V$ 's. Na curva 2 se observam os efeitos dos limites dos tap's de transformadores, e na curva 3 os limites de potência reativa das barras $P V$ 's juntamente com os dos tap's. Os respectivos PMC são os pontos "A", "B"e "C".

Destes resultados pode-se concluir que a inclusão dos limites no controle de potência reativa, ao contrário dos limites de controle de tap's, ocasionam uma grande redução no valor do PMC, i.e., no máximo carregamento ou na margem de carregamento do sistema. Deve se observar que em geral essas curvas não são previamente conhecidas. Também se observa que suas formas podem se alterar em conseqüência de alterações nas condições de operação do sistema de potência tais como:

- limites de potência reativa dos geradores e condensadores síncronos, ver figuras 1(b) e (c). Observe que enquanto as potências reativas geradas $\left(Q_{g 4}\right.$ e $\left.Q_{g 8}\right)$ nas barras de tensão controlada (barras PV) permanecem entre os seus respectivos limites, as respectivas magnitudes de tensão $\left(V_{4}\right.$ e $\left.V_{8}\right)$ permanecem com seus valores constantes. Quando os limites são atingidos e o tipo das barras alteradas para PQ, as magnitudes de tensão variam. No caso, começam a diminuir de valor em virtude do aumento do carregamento;

- limites de controle de tap's, ver figuras 1(d) e (e). Observe que enquanto o tap do transformador de número $8\left(t_{8}\right)$ permanece entre os seus respectivos limites mínimo e máximo $(0,95$ e 1,05$)$, a respectiva magnitude de tensão $\left(V_{5}\right)$ permanece com seu valor especificado. Ao atingir seu limite, a magnitude de tensão variará. $\mathrm{O}$ mesmo já não ocorre com o tap $\left(t_{51}\right)$ que controla a magnitude de tensão da barra $37\left(V_{37}\right)$, o qual permanece o tempo todo fixo em seu valor máximo;

- saídas de linhas de transmissão ou transformadores, ver figura 2, que representa o efeito da contingência da linha de transmissão situada entre as barras 69 e 75 do sistema IEEE-118.

Como se pode ver dessas curvas, a magnitude de tensão de uma barra pode permanecer constante ao longo de uma am-
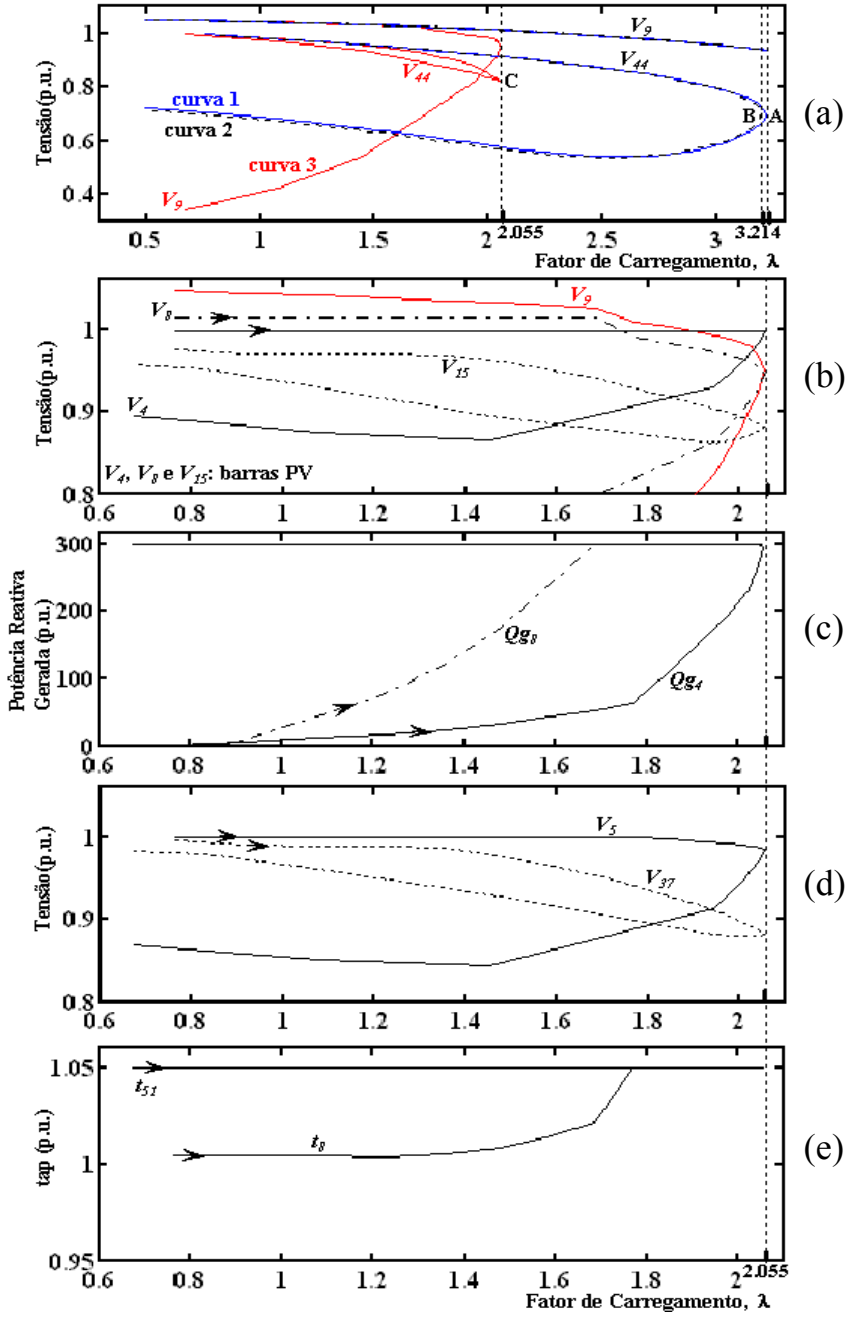

(c)

Figura 1: (a) Efeito dos limites sobre a curva P-V, (b) magnitudes de tensão de algumas barras de geração (barras PV), (c) potência reativa gerada pelas barras PV, (d) magnitudes de tensão de algumas barras controladas por tap, (e) variações dos tap's de alguns transformadores.

pla faixa da curva P-V e dessa forma, não poderia ser utilizada como parâmetro para se obter essa parte da curva P-V. Um outro aspecto importante é que uma barra de carga, por exemplo, a barra 9, cuja magnitude de tensão é apropriada para ser usada como parâmetro numa dada condição operativa (curva 3 da figura 1(a)), não o seria em outras condições, como no caso da curva 1 que desconsidera a atuação dos tap's (tap's mantidos fixos) e dos limites das potências reativas das barras $P V$ 's, ou, conforme se pode ver na figura 2, da condição de pós-contingência da linha de transmissão situada entre as barras 69 e 75 do sistema IEEE-118. O mesmo se pode afirmar com relação curvas $\mathrm{P}-\mathrm{V}$ de pré-contingência, das magnitudes de tensão das barras 75 e 118, apresentadas na figura 2. Como se pode ver dessas figuras, tanto o fator de 


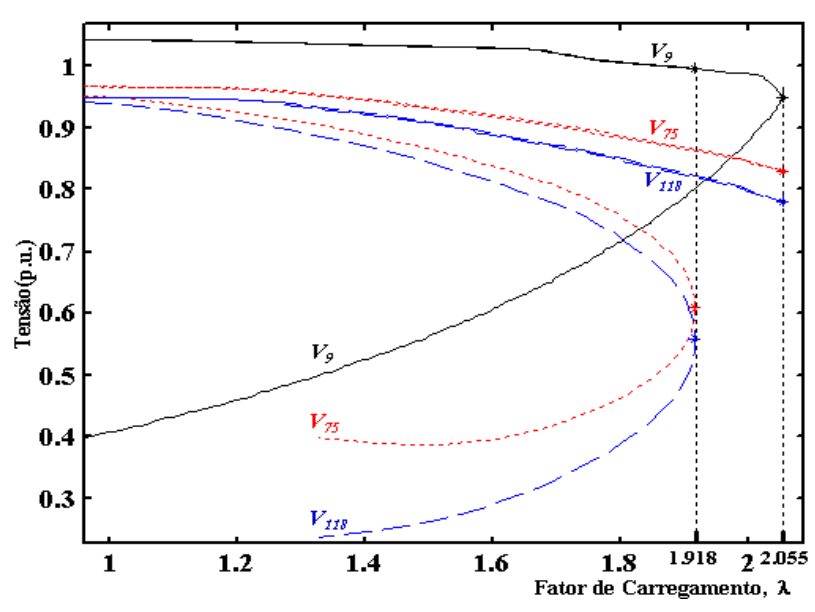

Figura 2: Curvas P-V para o caso base e para a contingência da linha de transmissão situada entre as barras 69 e 75 do sistema IEEE-118.

carregamento quanto a magnitude da tensão apresentam uma inversão simultânea na sua tendência de variação, i.e., os noses são coincidentes. Essa coincidência implica que a singularidade da matriz Jacobiana, a qual ocorre no PMC quando $\lambda$ é usado como parâmetro, é coincidente com a singularidade da matriz Jacobiana modificada quando a magnitude de tensão de uma dessas barras é utilizada como parâmetro (Alves et al., 2000).

Neste trabalho é apresentado um novo esquema de parametrização para o método da continuação que possibilita o traçado da curva P-V de um sistema de potência objetivando a obtenção do PMC e, subseqüentemente, a avaliação da margem de estabilidade de tensão. O objetivo foi o de obter uma técnica de parametrização geométrica que associasse a robustez com a simplicidade e a facilidade de interpretação. Assim, para a obtenção da solução do problema foi adicionada a equação de uma reta que passa por um ponto no plano formado pelas variáveis magnitude de tensão nodal e o fator de carregamento, podendo a reta ser mudada para outro ponto no mesmo plano, dependendo da curva P-V da barra em estudo. A mudança no valor do coeficiente angular é feita somente quando for necessário, i.e., ou quando o número de iterações excederem um determinado valor predefinido ou quando o método divergir. O coeficiente angular da reta é escolhido de forma a se obter um baixo número de iterações ao longo de todo o traçado da curva. O uso desta técnica amplia o grupo das variáveis de tensão que podem ser adotadas como parâmetro sem acarretar modificações na ordem da matriz Jacobiana do método proposto por Ajjarapu e Christy, (1992), e elimina a necessidade de troca de parâmetro ao longo do traçado da curva P-V. Este conjunto passa agora a incluir as barras cuja magnitude de tensão permanece constante ao longo de uma faixa da curva $\mathrm{P}-\mathrm{V}$, ou seja, barras de geração e as controladas por ajuste de tap sob carga, as quais antes não poderiam ser utilizadas como parâmetro para se obter essa parte da curva P-V (ver figuras 1(a), 1(b) e 1(d)). Também possibilita o uso da magnitude da tensão das barras cujos noses são coincidentes com o do fator de carregamento. Os resultados obtidos com o novo método para os sistemas testes do IEEE (14, 118 e 300 barras) mostram que as características de convergência do método de FC são melhoradas na região do PMC, e que este ponto pode ser determinado com a precisão desejada.

\section{O MÉTODO DA CONTINUAÇÃO}

$\mathrm{O}$ conjunto de equações de FC utilizado para representar o sistema elétrico de potência nos estudos de estabilidade estática de tensão é o seguinte:

$$
\begin{aligned}
& \mathbf{G}(\theta, \mathbf{V}, \lambda)=\mathbf{0} \text { ou } \\
& \mathbf{P}^{e s p}(\lambda)-\mathbf{P}(\theta, \mathbf{V})=\mathbf{0} \mathrm{e} \\
& \mathbf{Q}^{e s p}(\lambda)-\mathbf{Q}(\theta, \mathbf{V})=\mathbf{0} \\
& \mathbf{P}^{e s p}(\lambda)=\mathbf{P}_{\text {ger }}(\lambda)-\mathbf{P}_{\text {carga }}(\lambda) \mathrm{e} \\
& \mathbf{Q}^{\text {esp }}(\lambda)=\mathbf{Q}_{\text {ger }}-\mathbf{Q}_{\text {carga }}(\lambda) \\
& \mathbf{P}_{\text {carga }}(\lambda)=\lambda \mathbf{k}_{\text {pc }} \mathbf{P}_{\text {carga }}^{\text {sep }}, \\
& \mathbf{P}_{\text {ger }}(\lambda)=\lambda \mathbf{k}_{\text {pg }} \mathbf{P}_{\text {ger }}^{\text {esp }} \mathrm{e} \\
& \mathbf{Q}_{\text {carga }}(\lambda)=\lambda \mathbf{k}_{q c} \mathbf{Q}_{\text {carga }}^{\text {esp }}
\end{aligned}
$$

onde $\mathbf{G}$ é um vetor composto pelas equações dos balanços de potências ativa e reativa nodais, $\lambda$ é o fator de carregamento, V é o vetor das magnitudes das tensões nodais e $\theta$ é o vetor do ângulo das tensões nodais. $\mathbf{P}^{e s p}$ é o vetor da diferença entre as potências ativas gerada e consumida para as barras de carga $(P Q)$ e de geração $(P V)$ e $\mathbf{Q}^{e s p}$ é o vetor da diferença entre as potências reativas gerada e consumida para as barras de carga $P Q . \mathbf{P}_{c a r g a}^{e s p}, \mathbf{Q}_{c a r g a}^{e s p}$ e $\mathbf{P}_{g e r}^{e s p}$ são respectivamente os valores especificados no caso base $(\lambda=1)$ das potências ativa e reativa das barras $P Q$, e das potências ativa das barras $P V$. $\mathbf{k}_{p g}, \mathbf{k}_{p c}$ e $\mathbf{k}_{q c}$ são parâmetros prefixados usados para caracterizar um cenário de carga específico. Eles descrevem as taxas de variação de potência ativa $\left(\mathbf{P}_{\text {ger }}\right)$ nas barras de geração (barras $P V$ ), e das potências ativa $(\mathbf{P})$ e reativa $(\mathbf{Q})$ nas barras de carga (barras $P Q$ ). Assim, é possível realizar uma variação de carregamento individual, isto é, para cada barra do sistema, considerando para cada uma, um crescimento de carga com fatores de potência diferentes aos do caso base. Tradicionalmente, entretanto, assume-se que o aumento de carga de uma determinada área é feito com fator de potência constante e proporcional ao carregamento do caso base com modelo de carga de potência constante (nesse caso $\mathbf{k}_{p g}, \mathbf{k}_{p c} \mathrm{e}$ $\mathbf{k}_{q c}$ são todos iguais a um), visto que este fornece a condição operacional mais segura para o sistema (WSCC, 1998). Esta 
condição será adotada para todos os casos apresentados neste trabalho.

Uma vez definido um padrão de variação da carga e uma estratégia de despacho da geração, é necessário saber o quanto a demanda poderá aumentar antes que o sistema entre em colapso, ou seja, qual é a margem de carregamento (MC) para as condições preestabelecidas. Para isso realiza-se o traçado da curva P-V por meio de sucessivas soluções do sistema de equações (1) utilizando um FC e considerando um crescimento da carga na direção predefinida. Nesse procedimento, $\mathbf{P}^{e s p}$ e $\mathbf{Q}^{e s p}$ são as variáveis independentes, enquanto que as magnitudes de tensão $(\mathbf{V})$ e os ângulos de fase $(\theta)$, excetuando os da barra referência, são as variáveis dependentes. Com a inclusão de $\lambda$ como variável em (1) o sistema resultará em $n$ equações e $n+1$ incógnitas. Assim, qualquer uma das $n+1$ incógnitas pode ser definida como parâmetro. Quando $\lambda$ é usado como parâmetro o sistema de equações (1) passa a ter novamente $n$ equações e $n$ incógnitas. O seu valor é incrementando gradualmente, a partir do caso base $(\lambda=1)$, até um valor para o qual não mais se obtenha solução (o processo iterativo do FC não converge). Nesse ponto realiza-se um controle de passo que consiste numa simples redução no incremento de $\lambda$ e a solução de um novo FC a partir da última solução convergida. O PMC é considerado como sendo o último ponto convergido, após sucessivas repetições desse procedimento. Entretanto, conforme já comentado, a divergência do FC é conseqüência da singularidade da matriz $\mathbf{J}$ do sistema de equações (1) no PMC e, portanto, não será possível determiná-lo precisamente.

Em Alves et al. (2004) mostrou-se que diferentes valores de PMC eram obtidos quando do uso dos FC convencionais de Newton e desacoplado rápido. Além da incerteza a respeito do ponto obtido ser realmente o PMC, as sucessivas reduções no passo (incremento do parâmetro $\lambda$ ) pode resultar num processo computacional lento e oneroso quando comparados aos métodos de FCC. Diversos autores propuseram diferentes implementações dos conhecidos métodos da continuação para superar as dificuldades numéricas introduzidas pela singularidade da matriz $\mathbf{J}$ e possibilitar a determinação do PMC (Cañizares et al., 1992; Alves et al., 2000; Ajjarapu e Christy, 1992; Chiang et al., 1995). Entre os muitos métodos descritos na literatura, o mais amplamente utilizado consiste de quatro elementos básicos: um procedimento de parametrização, um passo preditor, um controle de passo e um passo corretor.

A partir da solução do sistema de equações (1) para o caso base $\left(\theta^{0}, \mathbf{V}^{0}, \lambda^{0}=1\right)$, um passo preditor é executado para encontrar uma estimativa para a próxima solução. Os preditores mais utilizados são o tangente e o secante. No preditor tangente a estimativa é encontrada dando um passo de tamanho apropriado na direção do vetor tangente à curva $\mathrm{P}-\mathrm{V}$, no ponto correspondente à solução atual (Ajjarapu e Christy, 1992). São utilizados dois métodos secantes (Seydel, 1994; Chiang et al., 1995): o de primeira ordem, que usa as soluções atual e anterior, para estimar a próxima, e o de ordem zero, que usa a solução atual e um incremento fixo no parâmetro $\left(\theta_{k}, V_{k}\right.$ oug $\lambda$, ego caso do método proposto $\alpha$ ) como uma estimativa para a próxima solução, esta técnica será adotada neste trabalho.

A parametrização fornece uma forma de identificar cada solução ao longo da trajetória a ser obtida. A parametrização local consiste na troca de parâmetro próximo ao PMC (Ajjarapu e Christy, 1992). A variável escolhida é aquela que apresentar a maior variação no preditor tangente, e no secante, a que apresentar a máxima variação relativa. A partir daí, $\lambda$ passa a ser tratado como variável dependente, enquanto que a variável escolhida passa a ser o novo parâmetro. O sistema de equações (1) permanece com $n$ equações e $n$ incógnitas.

Finalmente, após se efetuar a previsão, torna-se necessário realizar a correção da solução aproximada para se obter a solução final. Na maioria das vezes o ponto obtido pelo passo preditor está próximo da solução correta e assim, poucas iterações são necessárias no passo corretor para a obtenção da solução correta, dentro da precisão desejada. O método de Newton é o mais usado no passo corretor. Neste passo uma equação do tipo $y-y^{e s t}=0$, onde $y$ e $y^{e s t}$ correspondem à variável escolhida como parâmetro de continuação e seu respectivo valor estimado, obtido pelo passo preditor, pode ser acrescentada ao sistema de equações (1), ou o valor do parâmetro, como é feito no caso do preditor de ordem zero, pode ser simplesmente fixado em $y^{\text {est }}$.

\section{MÉTODO PROPOSTO}

No Fluxo de Carga Continuado Proposto (FCCP) é acrescentado ao sistema de equações (1), a equação da reta (figura 3) que passa por um ponto escolhido $\mathrm{O}\left(V_{k}^{0}, \lambda^{0}\right)$ no plano formado pelas variáveis magnitude de tensão nodal de uma barra $k$ qualquer $\left(V_{k}\right)$ e o fator de carregamento $(\lambda)$ :

$$
\begin{aligned}
& \mathbf{G}(\theta, \mathbf{V}, \lambda)=\mathbf{0} \\
& \mathbf{W}(\theta, \mathbf{V}, \lambda, \alpha)=\alpha\left(\lambda-\lambda^{0}\right)-\left(V_{k}-V_{k}^{0}\right)=0
\end{aligned}
$$

onde o parâmetro $\alpha$ é o coeficiente angular da reta. Como uma equação é adicionada, $\lambda$ pode ser tratado como uma variável dependente e $\alpha$ como uma variável independente, ou seja, escolhida como parâmetro da continuação (seu valor é prefixado). Assim, o número de incógnitas é igual ao de equações, isto é, a condição necessária para que se tenha solução é atendida, desde que a matriz tenha posto máximo, isto é, seja não singular. Observa-se que a prefixação do valor 


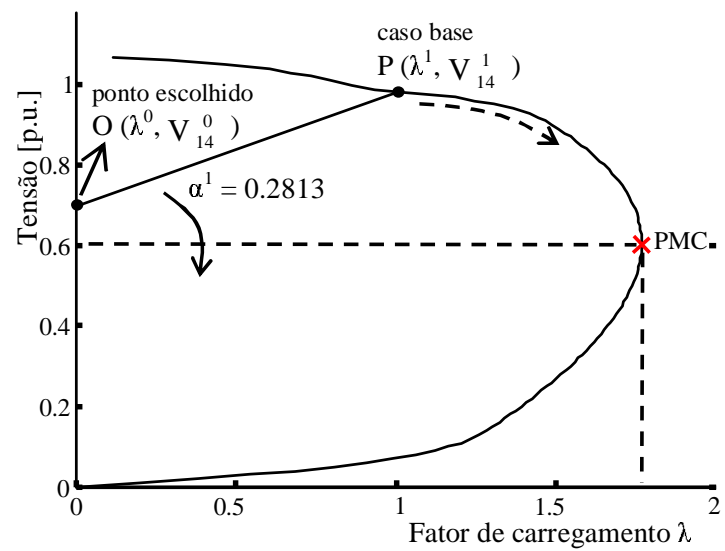

Figura 3: Reta inicial que passa por um ponto escolhido $\mathrm{O}$ $\left(\lambda^{0}, V^{0}\right)$ e o de caso base $\mathrm{P}\left(\lambda^{1}, V^{1}\right)$ no plano $\lambda \mathrm{V}$.

de $\alpha$ corresponde à técnica de previsão trivial ou polinomial modificada de ordem zero (Seydel, 1994). Com a solução do caso base $\left(\theta^{1}, \mathbf{V}^{1}\right.$ e $\left.\lambda^{1}\right)$, obtida com um FC onde $\lambda^{1}=1.0$, calcula-se o valor de $\alpha$ a partir do ponto inicial escolhido $\mathrm{O}$ $\left(\lambda^{0}, V_{k}^{0}\right)$ e dos seus respectivos valores obtidos no caso base $\mathrm{P}\left(\lambda^{1}, V_{k}^{1}\right)$ :

$$
\alpha^{1}=\left(V_{k}^{1}-V_{k}^{0}\right) /\left(\lambda^{1}-\lambda^{0}\right)
$$

A seguir, o FCCP é utilizado para calcular as demais soluções através dos sucessivos incrementos $(\Delta \alpha)$ no valor de $\alpha$. Para $\alpha=\alpha^{1}+\Delta \alpha$, a solução do sistema de equações (2) fornecerá o novo ponto de operação $\left(\theta^{2}, \mathbf{V}^{2}\right.$ e $\left.\lambda^{2}\right)$ correspondente a interseção da trajetória de soluções (curva P-V) com a reta cujo novo valor de coeficiente angular $\left(\alpha^{1}+\Delta \alpha\right)$ foi especificado. Para $\alpha=\alpha^{1}$, a solução convergida deverá resultar em $\lambda=1$. A expansão do sistema de equações (2) em série de Taylor, incluindo somente os termos de primeira ordem, considerando o valor prefixado no valor do parâmetro $\alpha$ calculado para o caso base, resulta em:

$$
\left[\begin{array}{cc}
-\mathbf{J} & -\mathbf{G}_{\lambda} \\
-\frac{\partial W}{\partial \mathbf{x}} & -\alpha
\end{array}\right]\left[\begin{array}{c}
\Delta \mathbf{x} \\
\Delta \lambda
\end{array}\right]=\mathbf{J}_{\mathbf{m}}\left[\begin{array}{c}
\Delta \mathbf{x} \\
\Delta \lambda
\end{array}\right]=\left[\begin{array}{c}
\Delta \mathbf{G} \\
\Delta W
\end{array}\right]
$$

onde $\mathbf{x}=\left[\theta^{T} \mathbf{V}^{T}\right]^{T}, \mathbf{J}$ é a matriz Jacobiana do FC, e $\mathbf{G}_{\lambda}$ corresponde à derivada de $\mathbf{G}$ em relação a $\lambda . \Delta \mathbf{G}$ e $\Delta W$ representam os fatores de correção (mismatches) das respectivas funções no sistema de equações (2). Deve-se observar que estes serão iguais a zero (ou praticamente nulos, isto é, inferior a tolerância adotada) para o caso base convergido.
Assim, somente $\Delta W$ será diferente de zero devido à variação de $\alpha$, i.e., devido ao seu incremento $\Delta \alpha$

\subsection{Procedimento Geral para a Mudança de Reta Durante o Traçado da Curva P-V}

Em função das análises realizadas definiu-se um procedimento geral para escolher o melhor feixe de retas a ser utilizado em cada região da curva P-V. Após muitos testes concluiu-se que o algoritmo a seguir é o mais robusto e o que exige a menor demanda em termos do número total de iterações necessária para o traçado da curva $\mathrm{P}-\mathrm{V}$. O procedimento geral adotado para o traçado da curva $\mathrm{P}-\mathrm{V}$ (observe na figura 4), pelo FCCP é o seguinte:

1. Obtenha o ponto "P"para o caso base utilizando o FC convencional e calcule por meio da equação (3) o correspondente valor do coeficiente angular da reta $\left(\alpha^{1}\right)$ que passa pelo ponto escolhido $\left(V_{k}^{0}=0,7\right.$ p.u., $\left.\lambda^{0}=0,0\right)$, ponto "O", e pelo ponto "P".

2. Obtenha os próximos pontos da curva P-V incrementando gradualmente (i.e., com um passo fixo, $\Delta \alpha=-0,05)$ o valor de $\alpha, \alpha^{i+1}=\alpha^{i}+\Delta \alpha ;$

3. Quando o FCCP não encontrar solução, retorne ao ponto anterior (ponto "E") e efetue uma redução no passo $(\Delta \alpha=-0,05 / 10)$;

4. Caso o FCCP divirja novamente, efetue a mudança das coordenadas do centro do feixe de retas para o ponto médio (ver detalhe na figura 4(b)) situado entre os dois últimos pontos obtidos, pontos "a"e "b", ou seja, [ $\left(V_{a}+\right.$ $\left.\left.V_{b}\right) / 2,\left(\lambda_{a}+\lambda_{b}\right) / 2\right]$ será as coordenadas do novo centro de feixe de retas denominado ponto médio. A seguir, considere a equação da reta que passa pelas coordenadas do novo centro de feixe de retas (ponto médio) e pelo último ponto convergido (ponto "b"). Observe que no caso do valor do $\lambda$ do ponto "b"ser maior do que o do ponto "a", o valor do passo deverá ser mantido em 0,005 , caso contrário, mantém-se o passo inicial de 0,05 ;

5. Quando o valor da magnitude da tensão do ponto atual (ponto "d"no detalhe da figura 4(b)) for maior que o do ponto anterior (ponto "c"), considera-se a equação da reta que passa pelas coordenadas do centro do feixe de retas inicial (ponto "O") e do ponto atual (ponto "d", do segundo feixe de retas) e completa-se o traçado da curva P-V (parte de baixo da curva P-V, figura 4(a)) com passos de 0,02 (observe que a troca do sinal se deve à mudança no sentido, que agora é anti-horário). 
O respectivo fluxograma pode ser visto no apêndice. As coordenadas iniciais do centro do feixe de retas, ponto "O", foram escolhidas de modo a possibilitar o traçado da curva $\mathrm{P}-\mathrm{V}$ de qualquer sistema desejado. Sua escolha foi baseada principalmente nos resultados apresentados durante a realização de vários testes considerando diversas magnitudes de tensão e vários sistemas. Inicialmente a escolha foi norteada pela constatação de que o uso das variáveis de tensão cuja magnitude da tensão permaneciam fixas no valor mínimo num trecho relativamente grande durante o traçado das curvas P$\mathrm{V}$ (ver figura 1), o valor a ser adotado inicialmente para a magnitude de tensão deveria ser inferior ao valor mínimo da faixa operativa normal de tensão adotada, no caso, inferior a 0,9 p.u.

Um outro fato que também foi levado em conta para a escolha das coordenadas iniciais do centro do feixe de retas foi o de que as magnitudes de tensão das barras críticas da maioria dos sistemas analisados (IEEE-14, 30, 57 e 300) são em torno de 0,7 p.u., o que, como será visto mais a frente (ver figura 6 para o caso do sistema IEEE-14), facilita o traçado da curva $\mathrm{P}-\mathrm{V}$, ou seja, não haverá necessidade de troca das coordenadas do centro do feixe de retas durante todo o traçado da curva. Conforme foi mostrado na figura 1, deve-se lembrar também que, em geral, é muito difícil identificar qual a magnitude de tensão é mais apropriada para ser usada para se obter todos os pontos da curva P-V, ou seja, no início dos testes não se conhece qual é a barra crítica do sistema em estudo, i.e., não se conhece a priori, a curvatura da trajetória de soluções (curva P-V). Assim, após a realização dos testes verificou-se que a adoção dos valores de 0,7 p.u. para tensão e de 0,0 para $\lambda$ como coordenadas iniciais do centro do feixe retas, não só conduziam ao sucesso no traçado completo da curva $\mathrm{P}-\mathrm{V}$ de praticamente todas as barras de todos os sistemas estudados, mas também, no geral, levavam ao melhor desempenho do método.

Por outro lado, observa-se que o uso das retas pertencentes ao feixe que passa pelo ponto médio é importante do ponto de vista da robustez do método, posto que seja necessário para eliminar as singularidades das matrizes Jacobianas. Observe também que a troca de feixe de retas implicará apenas na alteração do valor de $\alpha$ na matriz Jacobiana, ver equação (4), e não em mudanças na sua estrutura ou na criação de novos elementos.

\subsubsection{Análise da Variação do Mismatch Total de Potência para a Redução de Passo e Mu- dança das Coordenadas do Centro do Feixe de Retas}

É importante ressalvar que o critério adotado pelo método para a redução e mudança das coordenadas do centro do feixe de retas não se baseia apenas no número máximo de iterações adotado, que no caso é 10, mas também na análise do comportamento do mismatch total de potência. Na maioria dos casos as parametrizações resultam numa convergência mais rápida, entretanto algumas vezes isso não ocorre. Nestes casos a evolução dos respectivos mismatches indica a possibilidade de mau-condicionamento. Esse mismatch é definido como sendo a soma dos valores absolutos dos desbalanços de potência ativa e reativa.

Nas figuras 5(a) e (b) apresentam-se as evoluções dos mismatch para dois pontos da curva $\mathrm{P}-\mathrm{V}$ apresentada na figura 4. A evolução do mismatch para o ponto "E", figura 4(a), mostra que o processo iterativo convergiu em apenas 3 iterações para a tolerância de $10^{-4}$ p.u. adotada. Já a evolução do mismatch para o ponto seguinte (após o incremento de $-0,05$ no valor de $\alpha$ ), figura 5(b), mostra que o processo não converge em 10 iterações, e que na realidade o processo está divergindo. Voltando para a figura 4(a) verifica-se que nesse caso não há intersecção entre a reta tracejada e a curva P-V e assim, o problema na realidade não apresenta solução. Voltando ao ponto "E"e efetuando a redução do passo $(\Delta \alpha=-0,05 / 10)$ o problema volta a ter solução e com isso se obtém o ponto "F". O traçado da curva $\mathrm{P}-\mathrm{V}$ prossegue até que o problema se repete no ponto seguinte ao ponto "b", conforme se pode ver na figura 4(b).

A evolução dos mismatches correspondentes ao ponto "b"e ao ponto seguinte a este (reta tracejada), podem ser vistos nas figuras 5(c) e (d). Observe da evolução do mismatch para o ponto "b", figura 6(c), mostra que o processo iterativo convergiu em apenas 3 iterações para a tolerância de $10^{-4}$ p.u. adotada, enquanto que para o ponto seguinte (reta tracejada, após o incremento de $-0,005$ no valor de $\alpha$ ), o processo não diverge propriamente dito, mas apresenta um comportamento cíclico, cujo valor máximo reduz lentamente, porém voltando a crescer após 22 iterações, ver figura 5(e). Voltando para a figura 4(b) verifica-se, assim como no caso anterior, que o problema na realidade não apresenta solução, pois não há intersecção entre a reta e a curva P-V. Com base na análise desses casos verifica-se que após a quarta iteração já é possível se constatar esses comportamentos. Assim, optou-se por comparar, após a quarta iteração, os dois últimos valores do mismatch. Caso o último valor for menor que o penúltimo o processo continua iterando, caso contrário (se o último valor for maior que o penúltimo) finaliza o processo de iteração e retoma-se o traçado da curva $\mathrm{P}-\mathrm{V}$ a partir do ponto anterior. No primeiro caso (primeira divergência) a retomada do traçado é feita com o passo reduzido. No segundo caso, se efetua a mudança das coordenadas do centro do feixe retas para o ponto médio, ver figura 5(b).

Com o uso desta análise obteve-se uma redução das iterações, isto é, as mudanças sempre ocorreram antes de atingirse o número máximo de iterações estipulado, mostrando-se 


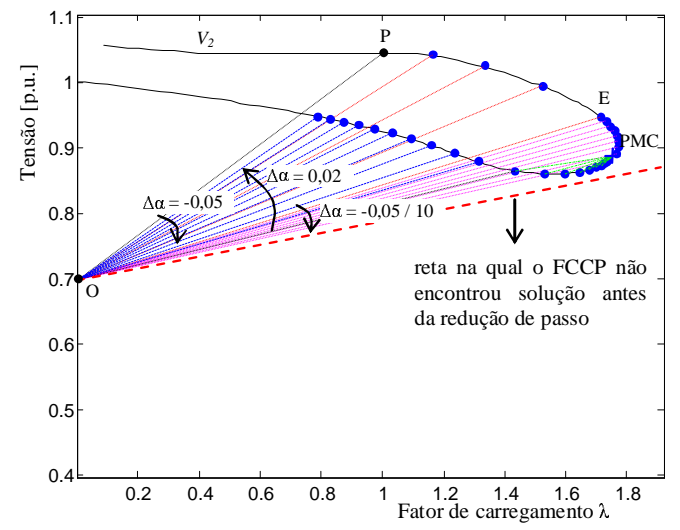

(a)

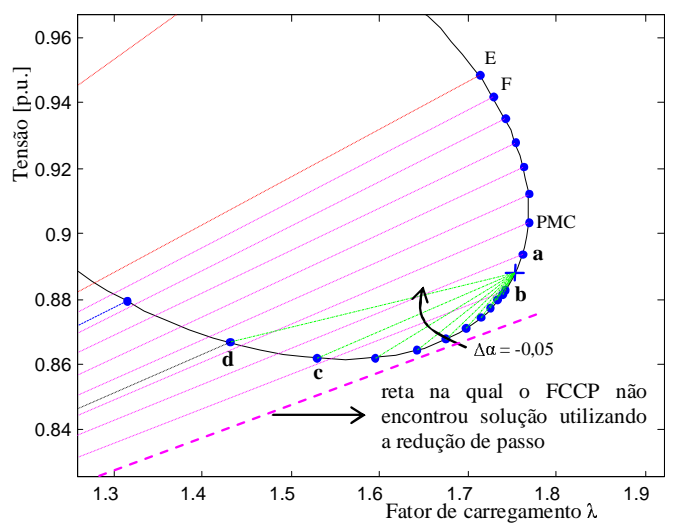

(b)

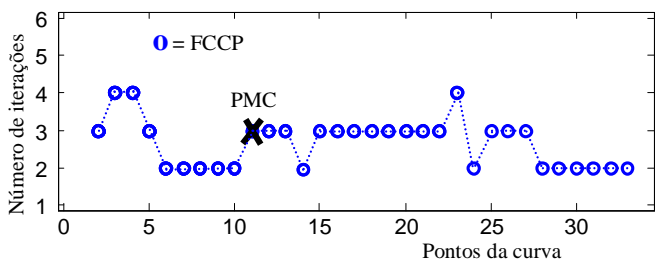

(c)

Figura 4: Desempenho do FCCP para o IEEE-14: (a) magnitude da tensão da barra de geração $2\left(V_{2}\right)$ em função de $\lambda$, (b) região do PMC ampliado, (c) número de iterações.

assim, mais vantajoso para o traçado da curva P-V. Por exemplo, para o sistema IEEE-14 a redução de passo e mudança de feixe ocorreu com o máximo de 6 iterações e nos sistemas, IEEE-118 e IEEE-300, com no máximo 7 iterações.

\section{RESULTADOS}

Para todos os testes realizados, a tolerância adotada para os mismatches foi de $10^{-4}$ p.u. O primeiro ponto de cada curva é obtido com o método de FC convencional. Os limites superiores e inferiores adotados para os tap's de transformadores foram 1,05 e 0,95 p.u. O ajuste do tap nos transformadores OLTC consiste da inclusão da posição dos tap's como variáveis dependentes, ao passo que as respectivas magnitudes das tensões das barras controladas são consideradas como variá-

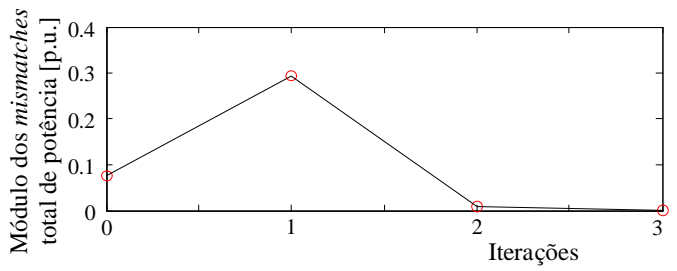

(a)
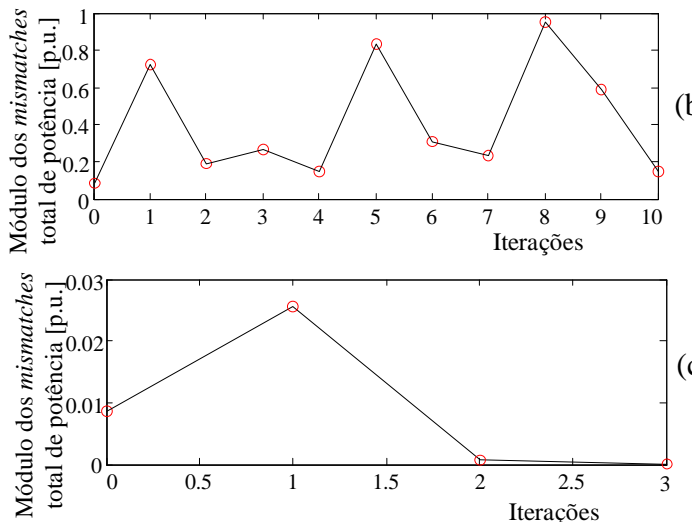

(c)
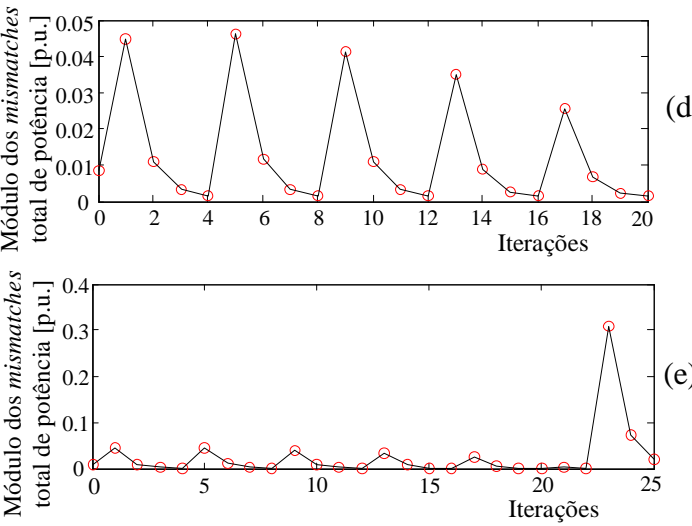

Figura 5: Desempenho do FCCP considerando a análise do módulo do mismatch total de potência: (a) para o ponto "E", (b) para o ponto seguinte ao ponto "E"mantendo o passo de -0,05 p.u., (c) para o ponto "b"com passo reduzido, (d) para o ponto seguinte ao ponto "b", (e) gráfico (d) considerando 25 iterações.

veis independentes (Peterson e Meyer, 1971; Monticelli A., 1983).

Os limites de potência reativa $(Q)$ nas barras $P V$ 's são os mesmos utilizados no método convencional de FC. Em cada iteração a geração de reativos de cada uma dessas barras é comparado com seus respectivos limites. No caso de violação, ela é alterada para tipo $P Q$. Estas barras podem voltar a ser $P V$ nas iterações futuras. As violações de limite de tap também são verificadas.

As cargas são modeladas como de potência constante e o parâmetro $\lambda$ é usado para simular incrementos de carga ativa e 
reativa, considerando fator de potência constante. Cada aumento de carga é seguido por um aumento de geração equivalente usando $\lambda$. O objetivo dos testes é mostrar as características do método proposto, considerando a influência dos limites de potência reativa $(Q)$ e de tap dos transformadores.

\subsection{Desempenho do Método Proposto para o Sistema IEEE-14}

A figura 6 mostra o desempenho do método proposto para o traçado da curva P-V do sistema IEEE-14. Na figura 6(a) é apresentado a tensão na barra crítica $\left(V_{14}\right)$ como função de $\lambda$, curva $\mathrm{P}-\mathrm{V}$, juntamente com as respectivas retas utilizadas. O ponto $\mathrm{P}\left(V_{14}^{1}=0,9813\right.$ p.u., $\left.\lambda^{1}=1\right)$ foi obtido com o FC convencional, e corresponde ao caso base. O valor correspondente do coeficiente angular da reta $\left(\alpha^{1}=0,2813\right)$ que passa pelo ponto escolhido $\left(V_{14}^{0}=0,7\right.$ p.u., $\left.\lambda^{0}=0,0\right)$ ponto $\mathrm{O}$, e pelo ponto $\mathrm{P}$, foi calculado por meio da equação (3). As demais retas foram obtidas considerando um passo $(\Delta \alpha)$ de $-0,05$ para $\alpha$.

A figura 6(b) apresenta o número de iterações gastas pelo FCCP para o traçado da curva P-V e também para o FCC parametrizado por tensão. A figura 6(c) apresenta a comparação entre os tempos de CPU gastos pelo FCCP e pelo FCC parametrizado pela magnitude de tensão da barra $14, V_{14}$. Os testes foram realizados em um processador Pentium 4 de $2.8 \mathrm{GHz}$. A diferença de tempo entre os métodos é praticamente desprezível, ou seja, os desempenhos dos métodos são praticamente os mesmos. Observe que nesse caso não foi necessário se efetuar a troca das coordenadas do centro do feixe de retas no FCCP, bem como do parâmetro no FCC parametrizado pela magnitude da tensão, durante todo o traçado da curva. Posteriormente, como será mostrado, o método proposto mostrar-se-á mais eficiente, uma vez que viabilizará, ao contrário do FCC parametrizado pela magnitude da tensão, o uso da magnitude de tensão de praticamente todas as barras.

\subsection{Desempenho do Método Proposto para o Sistema IEEE-118}

A figura 7 mostra o desempenho do FCCP para o traçado das curvas P-V do sistema IEEE-118, mostrando a robustez do método proposto. Na figura 7(a) encontra-se a curva $\mathrm{P}-\mathrm{V}$ completa da barra de carga 44 . O ponto de singularidade da matriz Jacobiana modificada utilizando a magnitude de tensão dessa barra como parâmetro da continuação coincide com o da matriz Jacobiana utilizando $\lambda$ como parâmetro, ou seja, com a singularidade da matriz J. Assim, essa variável não seria adequada para ser utilizada como parâmetro da continuação. Várias outras variáveis apresentam curvas $\mathrm{P}-\mathrm{V}$ com comportamento similar a esse, particularmente as mag-

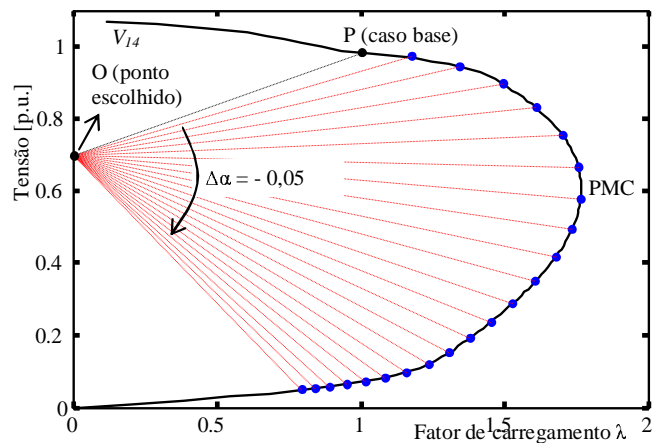

(a)

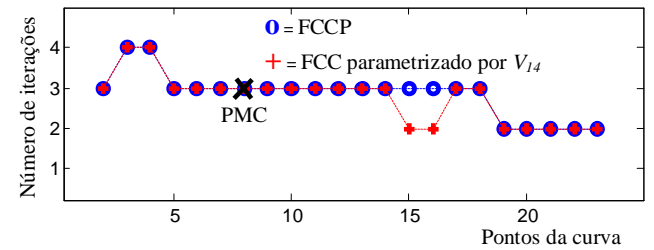

(b)

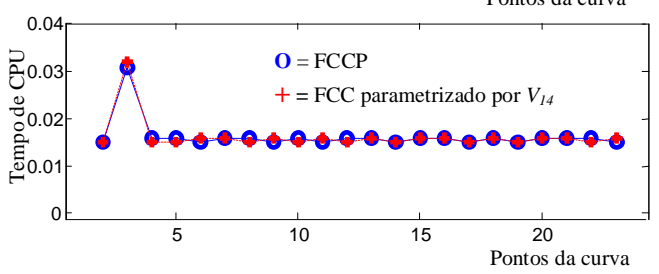

(c)

Figura 6: Desempenho do FCCP para o IEEE-14: (a) magnitude da tensão da barra crítica $\left(V_{14}\right)$ em função de $\lambda$, (b) número de iterações, (c) tempos de CPU para o FCCP e para o FCC parametrizado pela magnitude de tensão da barra 14 .

nitudes de tensão das barras de tensão controlada (barras $P V$ ) e das barras de carga (barras $P Q$ ) próximas a elas.

Conforme já se comentou anteriormente, muitas vezes as magnitudes de tensão das barras $P V$ permanecem constantes em um bom trecho da curva $\mathrm{P}-\mathrm{V}$ e assim, não costumam ser consideradas no conjunto daquelas que podem ser utilizadas como parâmetro da continuação.

Devido a estas limitações e ao fato de não se conhecer a priori o comportamento das curvas $\mathrm{P}-\mathrm{V}$, a escolha do parâmetro da continuação fica prejudicado, isto é, em muitos casos é necessário mudar algumas vezes de parâmetro antes de se obter sucesso na determinação do PMC. A seguir mostra-se que com o método proposto, até mesmo as variáveis cuja curva $\mathrm{P}-\mathrm{V}$ apresentam esse comportamento podem ser utilizadas. O processo inicia-se a partir do ponto "P". Na figura 7(b) se apresenta os pontos da parte estável (de cima) da curva P-V e que foram obtidos considerando os passos de 1 a 3 do procedimento geral. Quando o FCCP novamente não encontrar solução, toma-se às coordenadas dos dois últimos pontos da curva $\mathrm{P}-\mathrm{V}$, correspondentes às soluções do sistema de equações (2), e calculam-se as coordenadas do novo centro do feixe de retas, ponto médio na figura 7(c). 


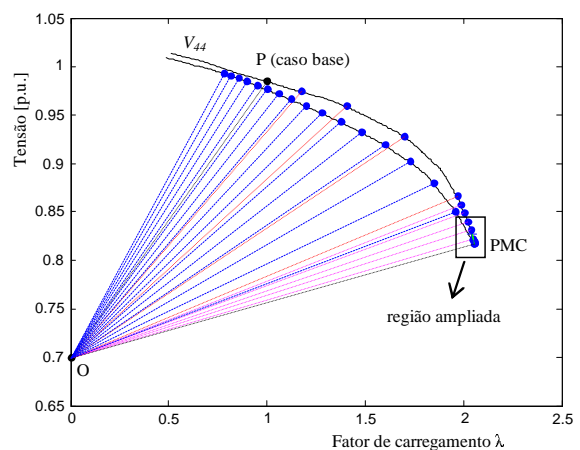

(a)

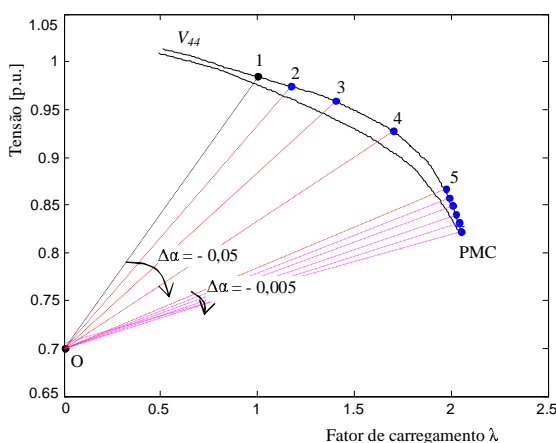

(b)
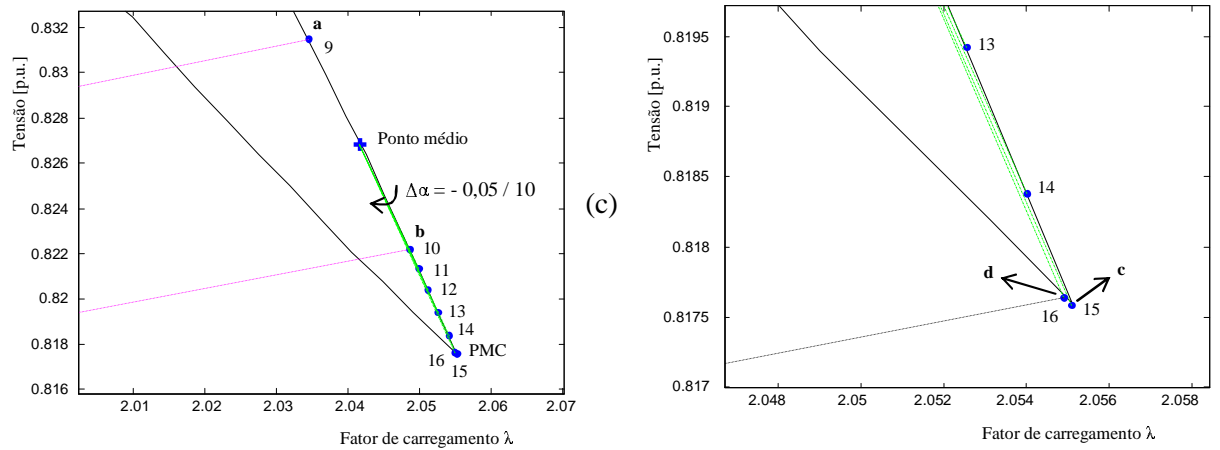

(d)
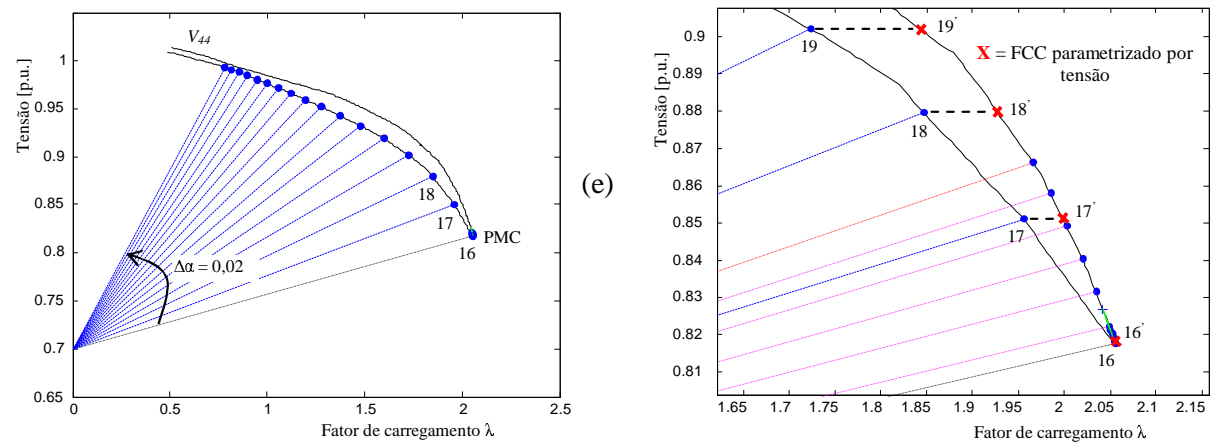

(f)

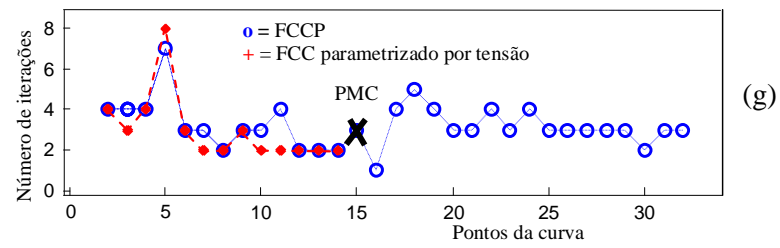

Figura 7: Desempenho do FCCP para o IEEE-118: (a) magnitude da tensão da barra de carga 44 em função de $\lambda$, curva P-V completa, (b) pontos da parte superior da curva P-V, (c) região do PMC ampliada, (d) últimos pontos do segundo feixe de retas, (e) pontos da parte inferior da curva P-V, (f) FCC parametrizado por tensão, (g) número de iterações.

Observe que o primeiro ponto da primeira reta do segundo feixe de retas é o último ponto "b"do primeiro feixe de retas, ponto 10 na figura $7(\mathrm{c})$. Utilizando os critérios do segundo feixe de retas e mantendo-se o mesmo passo $(-0,005)$ para $\alpha$, determinam-se os pontos pertencente à região do $\mathrm{PMC}$, figura 7(d). Quando o valor da magnitude da tensão do ponto atual for maior que a do ponto anterior, mudam-se novamente as coordenadas do centro do feixe de retas, de acordo com o passo 5 do procedimento geral. Assim, os pontos per- tencentes à parte inferior da curva P-V, figura 7(e), foram obtidos considerando a equação da reta que passa pelas coordenadas do centro do feixe de retas inicial (ponto "O") e do ponto atual (ponto "d", do segundo feixe de retas, neste caso o ponto 16). O passo adotado foi de 0,02 (observe que a troca do sinal se deve à mudança no sentido, que agora é antihorário), e assim completa-se o traçado da curva P-V (parte de baixo). 
As figuras 7(e) e (f) mostram que o FCCP não só obtém êxito em encontrar a solução, mas também permite a obtenção de pontos além do PMC (isto é, pontos da parte inferior da curva $\mathrm{P}-\mathrm{V})$ com um número reduzido de iterações, figura 7(f).

Com objetivo de comparar os desempenhos dos FCCP com o FCC parametrizado pela magnitude da tensão, os valores da magnitude da tensão da barra 44 foram armazenados durante o traçado da curva P-V utilizando o FCCP. Posteriormente, eles foram usados pelo FCC. Como se pode ver na figura 7(f), enquanto o FCCP fornece corretamente os pontos da parte inferior da curva $\mathrm{P}-\mathrm{V}$, o FCC parametrizado pela magnitude da tensão da barra 44 retorna pela parte superior da curva P-V (pontos de 16' a 19'), ou seja, não possibilita obter as soluções da parte inferior da curva P-V e assim, será necessário ponderar, da mesma forma que no caso do uso de $\lambda$ como parâmetro da continuação, se os problemas de não convergência são devidos a problemas numéricos ou a limitações físicas do sistema. Em geral, as diferenças não são óbvias.

Considere agora a curva P-V de uma barra PV cuja magnitude de tensão permanece constante ao longo de um trecho relativamente grande da curva $\mathrm{P}-\mathrm{V}$, no caso a barra de geração de número 46 do IEEE-118, veja a figura 8(a).

Observe que o método proposto obtém êxito no traçado de toda a curva P-V com um número reduzido de iterações, ver figura 8(c). Também é apresentado na figura 8(a), a curva $\mathrm{P}-\mathrm{V}$ da barra crítica (barra 13) em função de $\lambda$, com os respectivos pontos obtidos pelo uso da equação da reta situada no plano formado pela magnitude de tensão da barra 46 e o fator de carregamento $\lambda$. O objetivo é o de mostrar que os pontos obtidos realmente pertencem às partes superior e inferior da curva $\mathrm{P}-\mathrm{V}$, ou seja, que é possível a obtenção da curva P-V completa, apesar de essa curva apresentar um trecho para o qual há coincidência dos valores das magnitudes de tensão da parte superior e inferior da curva P-V.

A figura 9(a) mostra a curva P-V completa de uma outra barra de geração, a barra 76 do IEEE-118. Na figura 9(b) apresenta-se o número de iterações para cada ponto da curva $\mathrm{P}-\mathrm{V}$. Novamente, a figura 9(a) apresenta a curva P-V da barra 9, onde os pontos (círculos) pertencentes à curva da barra 9 foram obtidos utilizando a equação da reta situada no plano formado pela magnitude da tensão da barra 76 e o fator de carregamento.

Da curva P-V da barra 9 pode-se notar que na região do PMC, houve uma redução automática tanto no passo de $\lambda$ quanto no da magnitude de tensão, em consequiência direta da mudança das coordenadas do centro do feixe de retas (do ponto "O"para o ponto médio).
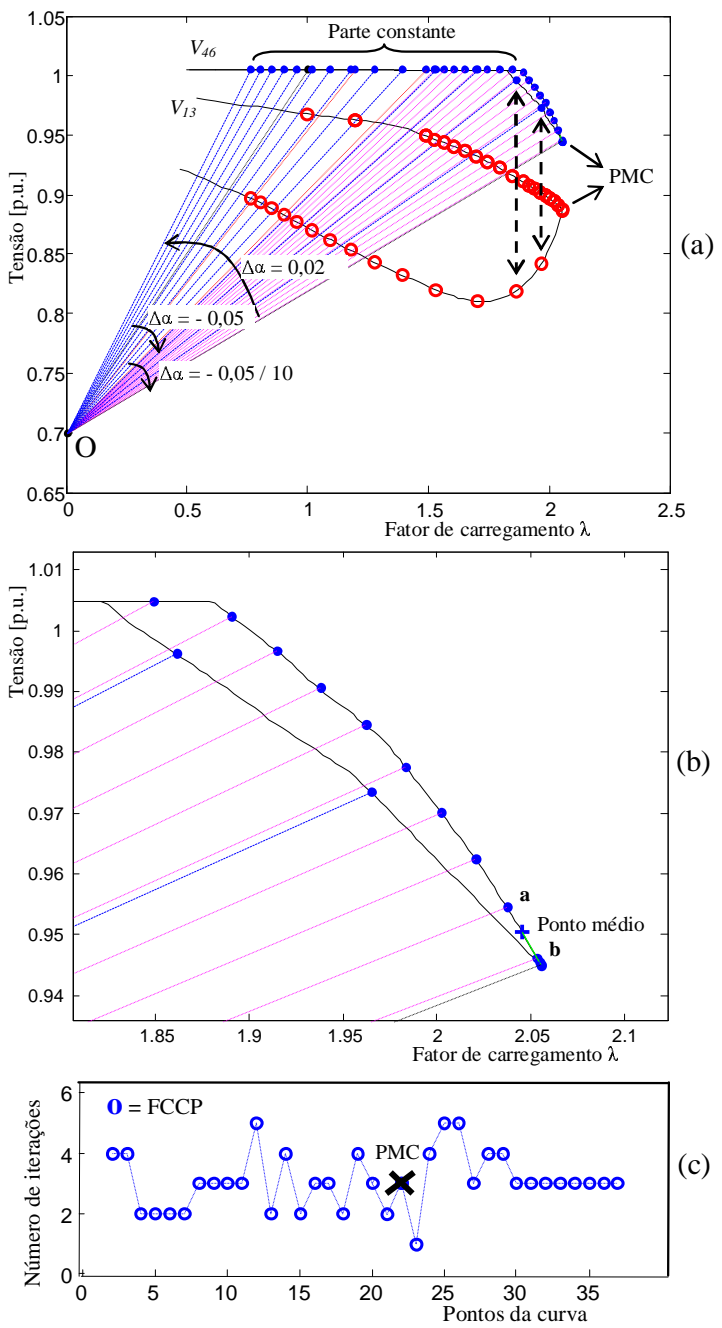

Figura 8: Desempenho do FCCP para o IEEE - 118: (a) magnitude da tensão da barra de geração 46 em função de $\lambda$, (b) região do PMC ampliada, (c) número de iterações.

\subsection{Desempenho do Método Proposto para o Sistema IEEE-300}

A figura 10 mostra o desempenho FCCP para o traçado das curvas P-V do sistema IEEE-300, mostrando a robustez do método proposto para um sistema de maior porte, onde será mostrado o desempenho do método proposto considerando as magnitudes de tensão da barra crítica 526, da barra 15 , e da barra PV de número 63, figuras 10, 11 e 12, respectivamente.

Da figura 10(a) se pode observar que no caso da barra crítica (barra 526), o algoritmo ficou restrito apenas aos passos $1 \mathrm{e}$ 2 do procedimento geral apresentado no item 3.1. A figura 10(b) mostra o número de iteração gasto pelo método proposto e pelo método do FCC parametrizado por tensão, de onde se pode notar que os desempenhos (número de itera- 


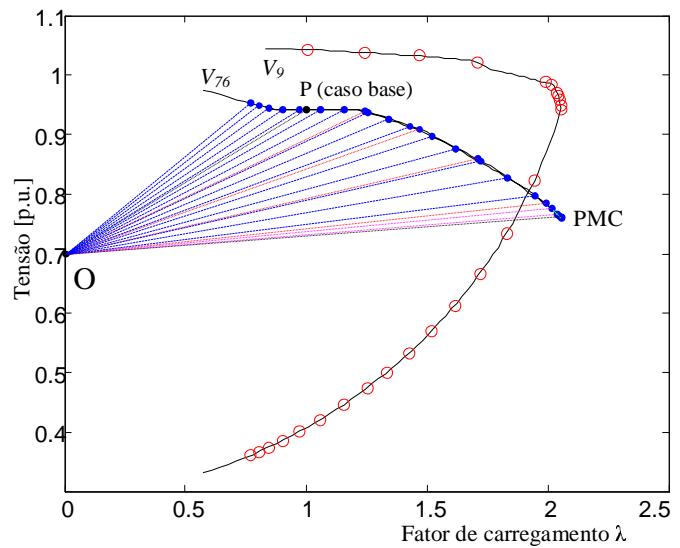

(a)

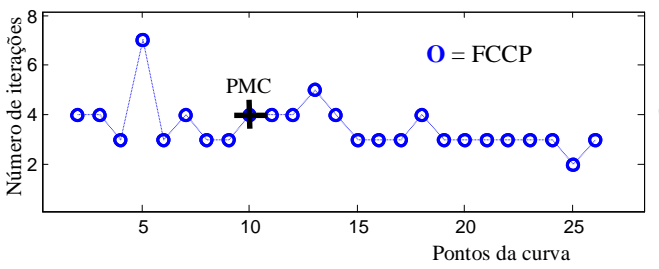

(b)

Figura 9: Desempenho do FCCP para o IEEE-118: (a) magnitude da tensão da barra de geração 76 em função de $\lambda$, curva P-V completa, (b) número de iterações.

ções) de ambos os métodos foram praticamente os mesmos.

A figura 10(c) apresenta a comparação entre os tempos de CPU gastos pelo FCCP e pelo FCC parametrizado pela magnitude de tensão da barra $526, V_{526}$. Os testes foram realizados em um processador Pentium 4 de $2.8 \mathrm{GHz}$. Novamente aqui, se pode considerar que a diferença de tempo entre os métodos é praticamente desprezível, ou seja, que os desempenhos dos métodos são praticamente os mesmos.

Já a figura 11 apresenta a aplicação do FCCP considerando a tensão da barra de carga 15 do sistema IEEE-300. A escolha da magnitude de tensão dessa barra se deve, conforme já comentado, ao fato de sua curva $\mathrm{P}-\mathrm{V}$ corresponder àqueles casos em que tanto o fator de carregamento quanto a magnitude da tensão apresentam uma inversão simultânea na sua tendência de variação, i.e., os noses são coincidentes, ou seja, há coincidência da singularidade de ambas as matrizes Jacobiana modificadas. Na figura 11(a) também é apresentado a curva P-V da barra crítica, barra 526, em função de $\lambda$, com os respectivos pontos (círculos) obtidos pelo uso da equação da reta situada no plano $\lambda \mathrm{V}$ (fator de carregamento $\lambda$ e magnitude de tensão da barra 15). Novamente, o objetivo é o de mostrar que os pontos obtidos realmente pertencem às partes superior e inferior da curva P-V. Os detalhes da aplicação do procedimento geral podem ser vistos nas figuras 11(b) e (c). As coordenadas do centro do feixe de retas inicial (ponto "O") são as mesmas, ou seja, $V_{526}^{0}=0,7$ p.u., $\left.\lambda^{0}=0,0\right)$, entretanto, as do ponto "P", denominado caso base e obtidas por

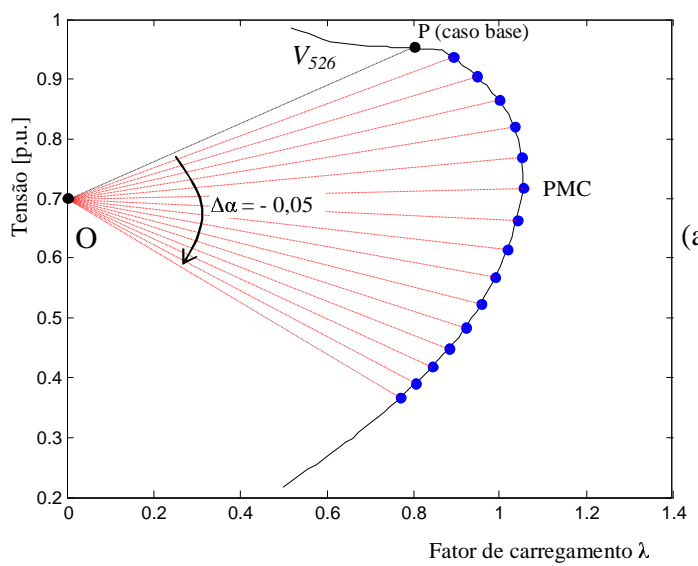

(a)
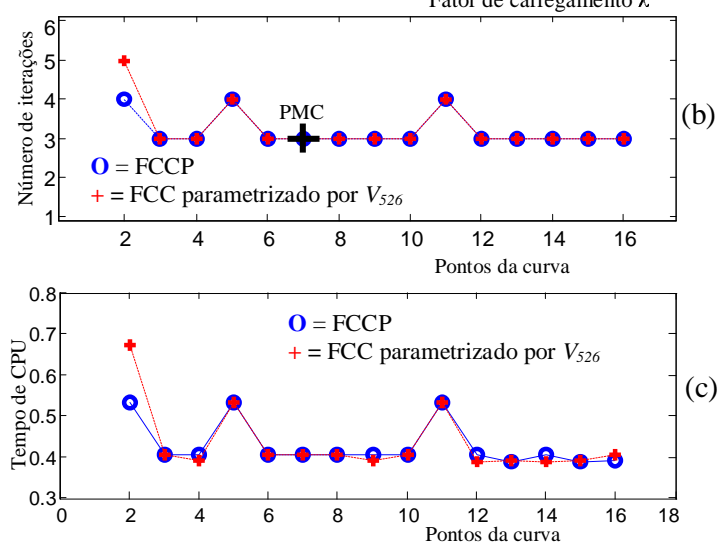

Figura 10: Desempenho do FCCP para o IEEE-300: (a) magnitude da tensão da barra de carga crítica $526\left(V_{526}\right)$ em função de $\lambda$, (b) número de iterações, (c) tempos de CPU para o FCCP e para o FCC parametrizado pela magnitude de tensão da barra 526.

um FC convencional, correspondem ao valor de $\lambda$ de 0,8 . Esse valor foi escolhido apenas para uma melhor visualização, pois o valor fator de carregamento do PMC dessas curvas estão em torno de 1,05, ou seja, muito próximo de 1,0.

A figura 11(d) apresenta o número de iterações do FCCP e o FCC parametrizado pela magnitude de tensão da barra 15, pode-se notar que até nas proximidades do PMC, os desempenhos são praticamente os mesmos. Observe, entretanto, que o FCC parametrizado pela magnitude de tensão não apresenta resultados nem para o PMC, nem para os pontos da parte de baixo da curva $\mathrm{P}-\mathrm{V}$, em virtude da singularidade da matriz Jacobiana modificada.

Uma outra curva P-V traçada é a da barra de geração 63. A escolha da magnitude de tensão dessa barra se deve, conforme já comentado, ao fato de sua curva $\mathrm{P}-\mathrm{V}$ corresponder àqueles casos em que além da magnitude de tensão da barra permanecer constante ao longo de uma faixa da curva P-V, e dessa forma não pode ser utilizada como parâmetro para 


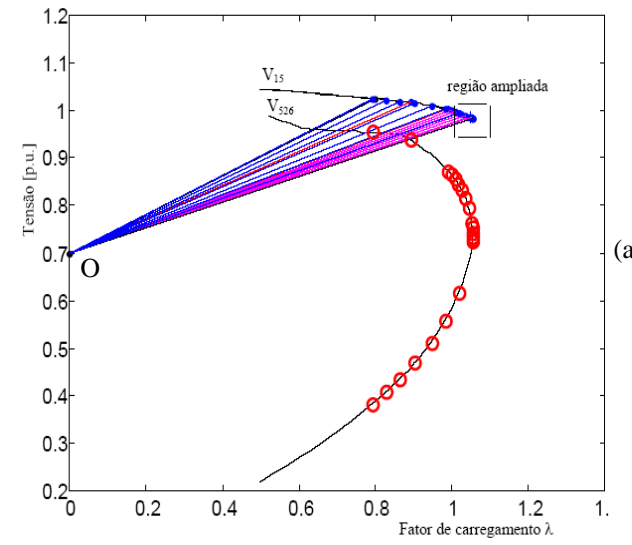

(a)
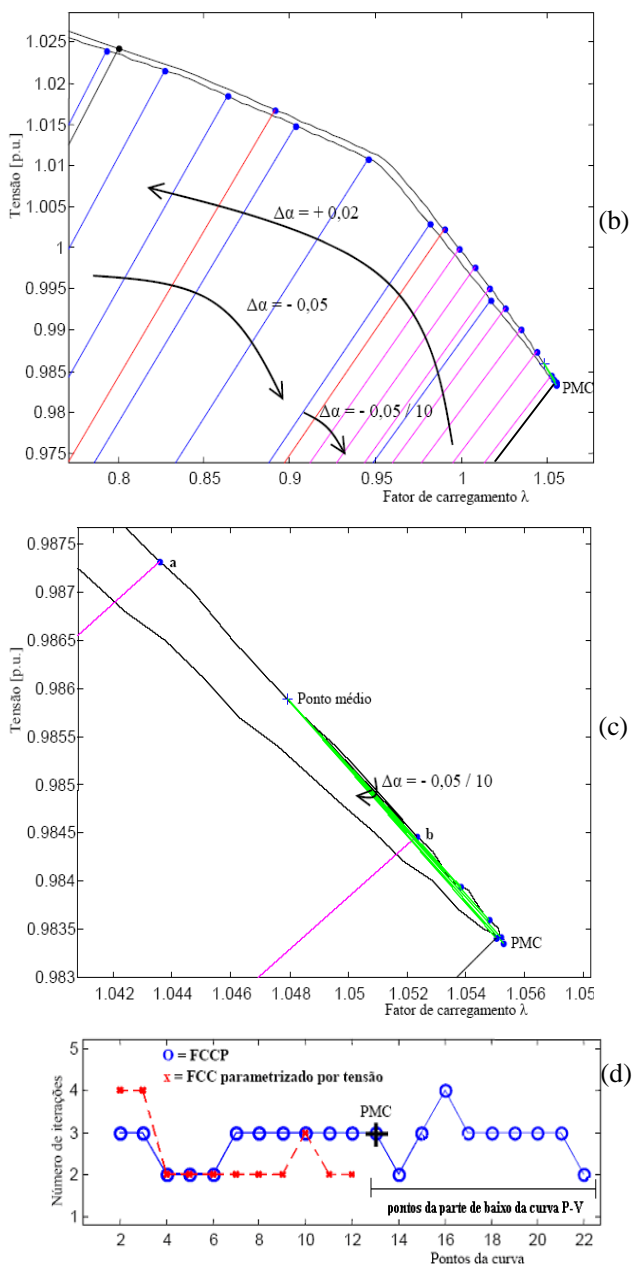

Figura 11: Desempenho do FCCP para o IEEE-300: (a) magnitude da tensão da barra de carga 15 em função de $\lambda$, (b) região estável e instável, (c) região do PMC ampliada, (d) número de iterações.

se obter essa parte da curva $\mathrm{P}-\mathrm{V}$, tanto o fator de carregamento quanto a magnitude da tensão apresentam uma inversão simultânea na sua tendência de variação, i.e., os noses
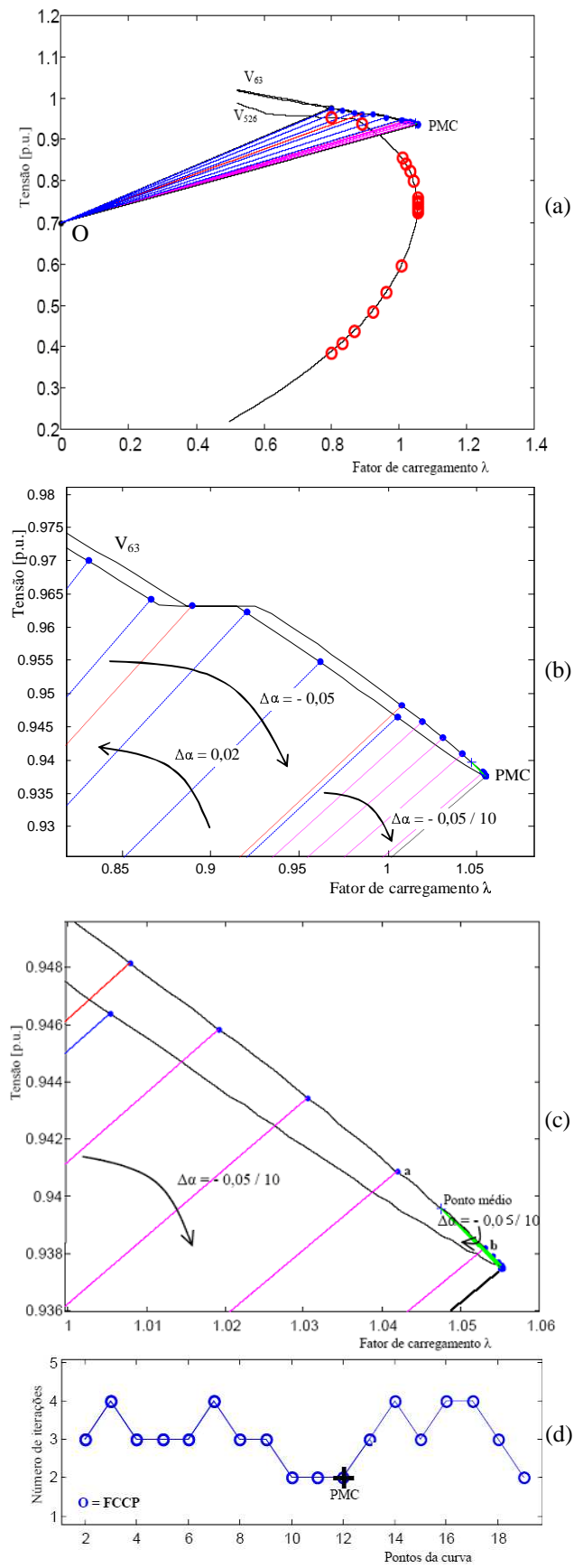

Figura 12: Desempenho do FCCP para o IEEE-300: (a) magnitude da tensão da barra de geração 63 em função de $\lambda$, (b) parte da curva ampliada, (c) região do PMC ampliada, (d) número de iterações.

são coincidentes.

Como se pode observar na figura 12, o método proposto apresenta um excelente desempenho durante todo o traçado da curva P-V. Novamente, a figura 12(a) apresenta a curva P-V 
da barra crítica 526, onde os respectivos pontos (círculos) foram obtidos pelo uso da equação da reta situada no plano $\lambda \mathrm{V}$ (fator de carregamento $\lambda$ e magnitude de tensão da barra 63).

De acordo com os resultados obtidos, o método apresenta um bom desempenho ao longo de todo o traçado da curva P-V, ver figura 12(b). Na vizinhança do PMC o método convencional de FC apresenta dificuldades numéricas, enquanto que o método proposto não só obtém êxito em encontrar a solução, mas também permite a obtenção de pontos além do PMC (isto é, pontos da parte inferior da curva P-V, figura 12(c)) com um número reduzido de iterações, figura 12(d).

\section{CONCLUSÕES}

O trabalho apresenta um novo esquema de parametrização que possibilita o traçado completo das curvas $\mathrm{P}-\mathrm{V}$ baseado em simples modificações do método de Newton, enquanto se preserva suas vantagens características. O método proposto não só obtêm êxito em encontrar, com a precisão desejada, as soluções na região do PMC e no próprio ponto, mas também permite a obtenção de soluções além deste (isto é, pontos da parte inferior da curva $\mathrm{P}-\mathrm{V}$ ) com um número baixo de iterações. Isto se tornou possível em virtude da remoção da singularidade da matriz Jacobiana no PMC. A remoção da singularidade foi obtida através do acréscimo de uma equação de reta no plano formado pela magnitude da tensão nodal de uma barra $k$ qualquer e o fator de carregamento $\lambda$.

De acordo com os resultados alcançados, o método apresentou um excelente desempenho na vizinhança do PMC. Uma outra vantagem importante acrescida pelo uso deste novo método foi à ampliação do conjunto de variáveis de tensão que podem ser adotadas como parâmetro da continuação. Este conjunto passa agora a incluir as barras cuja magnitude de tensão permanece constante ao longo de uma faixa da curva $\mathrm{P}-\mathrm{V}$, e dessa forma não pode ser utilizada como parâmetro para se obter essa parte da curva P-V. Também inclui as barras cuja magnitude da tensão apresenta uma inversão na sua tendência de variação simultaneamente com o fator de carregamento, i.e., os noses são coincidentes, ou seja, há coincidência da singularidade de ambas as matrizes Jacobianas no PMC (Alves et al., 2000; Ajjarapu e Christy, 1992).

Observa-se que em vários métodos existentes na literatura pode ser necessário se efetuar a mudança de parâmetro ao longo do traçado da curva $\mathrm{P}-\mathrm{V}$, o que poderá acarretar mudanças na estrutura da matriz Jacobiana modificada. No caso do método proposto, ao contrário do proposto por Ajjarapu e Christy, 1992, não se necessita realizar a troca de parâmetro ao longo de todo o traçado da curva $\mathrm{P}-\mathrm{V}$, sendo que algumas vezes apenas se faz uma mudança de coordenadas do centro do feixe de retas, o que, da mesma forma que o proposto por Ajjarapu e Christy (1992), também não implicará em mu- danças na estrutura da nova matriz, mas apenas do valor do elemento correspondente a derivada da equação de reta $(W)$ em relação ao fator de carregamento $(\lambda)$, ou seja, no valor do coeficiente angular da reta $(\alpha)$.

É importante ressalvar também a inclusão da análise do comportamento do mismatch total de potência no critério adotado pelo método para a redução do passo e mudança das coordenadas do centro do feixe de retas, o qual anteriormente era baseado apenas no número máximo de iterações. Mostrou-se que a evolução do mismatch indica a possibilidade de maucondicionamento. Com o uso desta análise obteve-se uma redução das iterações, isto é, as mudanças sempre ocorreram antes de atingir-se o número máximo de iterações estipulado, mostrando-se assim, mais vantajoso para o traçado da curva P-V.

\section{AGRADECIMENTOS}

Agradecemos à FAPESP e ao CNPq pelo apoio financeiro.

\section{REFERÊNCIAS}

Ajjarapu, V. e Christy, C., (1992). The Continuation Power Flow: a Tool for Steady State Voltage Stability Analysis, IEEE Trans. on Power Systems, v. 7, n. 1, February, p. 416-423.

Alves, D. A., (2000). Obtenção do Ponto de Máximo Carregamento em Sistemas Elétricos de Potência Utilizando Novas Técnicas de Parametrização para o Método de Continuação. Tese de doutorado. DSCE/FEEC. Universidade Estadual de Campinas.

Alves, D. A., da Silva, L. C. P., Castro, C. A., and da Costa, V. F., (2004). "Parameterized fast decoupled power flow methods for obtaining the maximum loading point of power systems-part-I: mathematical modeling", Electric Power Systems Research, v. 69, p. 93-104.

Alves, D. A.; da Silva, L. C. P.; Castro, C. A. e da Costa , V. F., (2000). Continuation Load Flow Method Parameterized by Power Losses, Proceedings of the IEEE Power Engineering Society Winter Meeting 2000, Singapore, January.

Cañizares, C. A.; Alvarado, F. L.; DeMarco, C. L.; Dobson, I. e Long, W. F., (1992). Point of Collapse Methods Applied to AC/DC Power Systems, IEEE Trans. on Power Systems, v. 7, n. 2, May, p. 673-683.

Chen J. F. e W.M. Wang (1997). Stability Limit and Uniqueness of Voltage Solutions for Radial Power Networks, Electric Machine and Power Systems, v. 25, p. 247-261. 
Chiang, H. D.; Flueck, A.; Shah, K. S. e Balu, N., (1995). CPFLOW: A Practical Tool for Tracing Power System Steady State Stationary Behavior Due to Load and Generation Variations, IEEE Trans. on Power Systems, v. 10, n. 2, May, p. 623-634.

FTCT-Força Tarefa "Colapso de Tensão" (1999). Critérios e Metodologias Estabelecidos no âmbito da Força - Tarefa "Colapso de Tensão" do GTAD / SCEL / GCOI para Estudos de Estabilidade de Tensão nos Sistemas Interligados Norte/Nordeste, Sul/Sudeste e Norte/Sul Brasileiros, XV SNPTEE, Foz do Iguaçu, PR, Brasil.

Garbelini, E.; Bonini, A. N.; Alves, D. A.; Righeto, E.; Silva, L. C. P.; Castro, C. A., (2005). Fluxo de Carga Continuado: Uma Nova Técnica de Parametrização Geométrica. $6^{\circ}$ Latin-American congress: Electricity Generation and Transmission, Mar del Plata, Argentina.

Iba, K.; Suzuki, H.; Egawa, M. e Watanabe, T., (1991). Calculation of Critical Loading Condition with Nose Curve Using Homotopy Continuation Method, IEEE Trans. on Power Systems, v. 6, n. 2, May, p. 585-593.

Monticelli A., (1983). Fluxo de Carga em Redes de Energia Elétrica, Edgard Blücher, São Paulo, 164p.

Peterson N. M. e W.S. Meyer (1971), Automatic Adjustment of Transformer and Phase-Shifter Taps in the Newton Power Flow, IEEE Trans. on PAS, Vol. PAS-90, $\mathrm{N}^{o} 1$, February, pp. 103-108.

Overbye T. J. e R. P. Klump (1996). Effective calculation of power systems low-voltage solutions. IEEE Trans. on Power Systems, Vol. 11, No. 1, pp. 75-82.

Seydel, R., (1994). From Equilibrium to Chaos: Practical Bifurcation and Stability Analysis, $2^{a}$ ed., Springer Verlag, New York, 407p.

Yorino N., S. Harada, e H. Cheng (1997). A Method to Approximate a Closest Loadability Limit Using Multiple Load Flow Solutions. IEEE Transactions on Power Systems, Vol. 12, No. 1, pp. 424-429.

WSCC-Reactive Power Reserve Work Group (RRWG) (1998). Final Report, Voltage Stability Criteria, Undervoltage Load Shedding Strategy, and Reactive Power Reserve Monitoring Methodology, May, 154p.

\section{APÊNDICE}

A figura 13 apresenta o fluxograma correspondente ao procedimento geral apresentado no item 3.1. Na figura, i corresponde ao número de pontos da curva $\mathrm{P}-\mathrm{V}$, e $\mathrm{k}$ corresponde a um contador que indica quando deverá ser efetuado a redução de passo ou a mudança das coordenadas do centro do feixe de retas. 


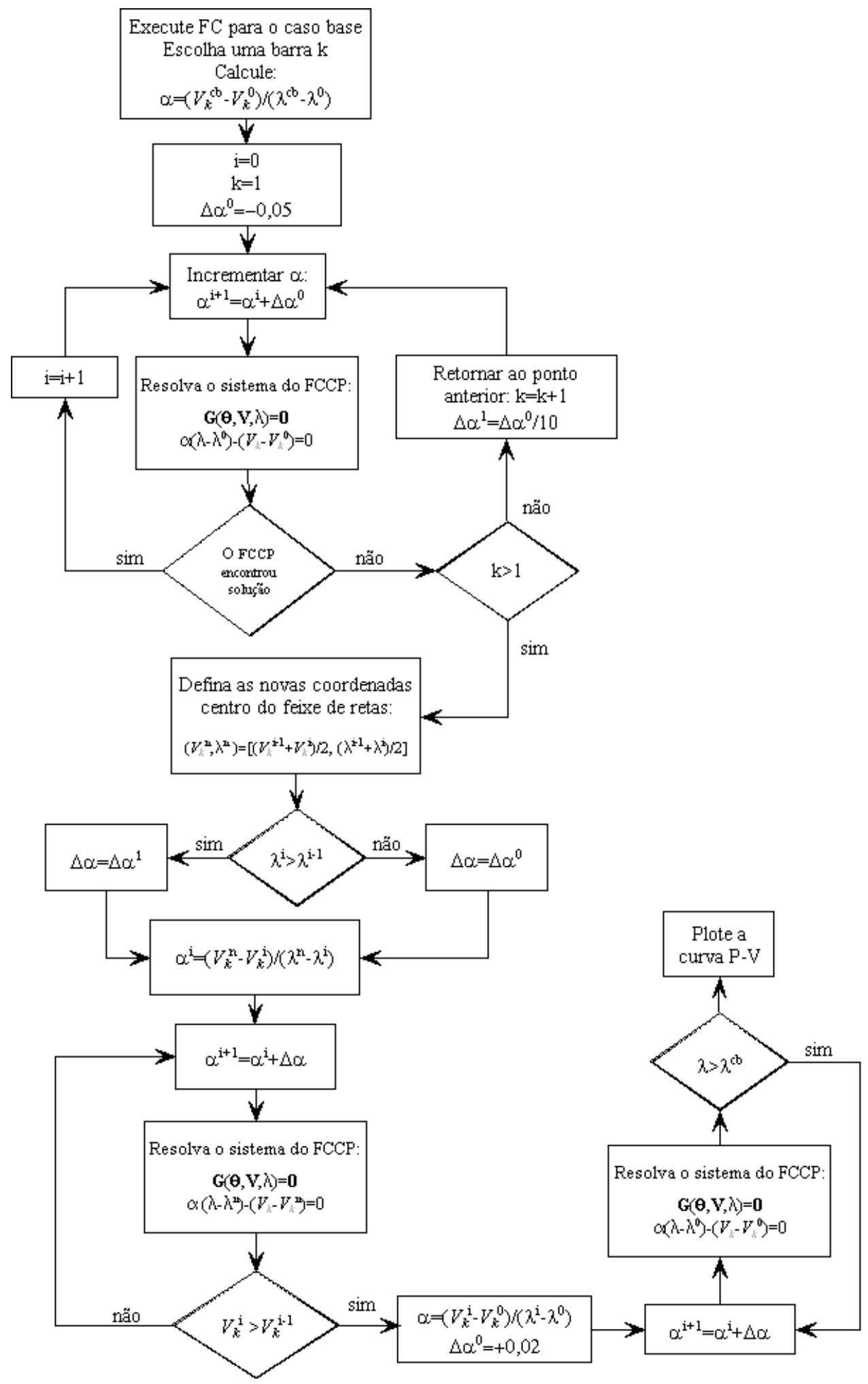

Figura 13: Fluxograma do FCCP. 
